\title{
Preservation of perfectness and acyclicity: Berrick and Casacuberta's universal acyclic space localized at a set of primes
}

\author{
José L. Rodríguez, Jérôme Scherer, And Antonio Viruel *
}

\begin{abstract}
In this paper we answer negatively a question posed by Casacuberta, Farjoun, and Libman about the preservation of perfect groups under localization functors. Indeed, we show that a certain P-localization of Berrick and Casacuberta's universal acyclic group is not perfect. We also investigate under which conditions perfectness is preserved: For instance, we show that if the localization of a perfect group is finite then it is perfect.
\end{abstract}

2000 Mathematics Subject Classification: 55P60, 20F38, 20J15, 20E26.

\section{Introduction}

The question of whether or not perfect groups are preserved under arbitrary group localization functors goes back to Farjoun and was raised simultaneously in [Li] and [Ca]. The close link between perfect groups and ordinary homology provides the topological motivation. Indeed if $X$ is a space with perfect fundamental group $H$, then of course $H_{1}(X ; \mathbb{Z})=0$ by the Hurewicz Theorem. One wonders next what happens with the localizations of $X$. Is it true that their first integral homology groups are still trivial? Likewise what can be said about the localizations of an acyclic space? A first step is to analyze

*José L. Rodríguez was partially supported by DGIMCYT grant BFM2001-2031, and EU grant nr HPRN-CT-1999-0011, J. Scherer by the program Ramón y Cajal, MCYT (Spain), and A. Viruel by MCYT grant BFM2001-1825, and EU grant nr HPRN-CT-1999-0011. 
the effect of localization functors in the category of groups, since any such functor yields a homotopical localization by applying the classifying space construction.

A positive result is Theorem 2.4 which claims that if $G$ is a perfect group and its localization is known to be finite then it is automatically perfect. Therefore any example of a non-perfect localization of a perfect group should be an infinite group. The reader which is not familiar with the general theory of localization should not worry since we will mostly deal with standard $P$-localization, where $P$ is a set of primes. The functor $L_{P}$ associates in a universal way to any group $G$ a morphism $G \rightarrow L_{P} G$ from $G$ to a uniquely $p$-divisible group for any prime number $p \notin P$. We ask now:

Question: If $G$ is a perfect group, does it imply that so is $L_{P} G$ ?

We have seen that we should look for large groups if we want to answer negatively to this question. Berrick and Casacuberta have precisely constructed in [BC] a universal acyclic space $B \mathcal{F}=K(\mathcal{F}, 1)$, whose associated nullification functor is equivalent to Quillen's plus construction (cf. [Dr, 1.A.4] for a definition of nullification). The fundamental group $\mathcal{F}$ is a free product of an uncountable set of locally free perfect groups and is a universal "generator" of all perfect groups in the sense that the evaluation map

$$
\mathcal{F} \times \operatorname{Hom}(\mathcal{F}, G) \stackrel{\text { ev }}{\longrightarrow} G
$$

given by ev $(x, \psi)=\psi(x)$ is surjective for every perfect group $G$. In particular for any acyclic group $G$ the cofibre of the evaluation map $\bigvee_{[B \mathcal{F}, B G]} B \mathcal{F} \rightarrow B G$ is simply connected and acyclic, thus contractible. By Chachólski's result [Ch, Theorem 20.9] it means that the cellularization $C W_{B \mathcal{F}} B G$ is $B G$, i.e. $B G$ can be constructed as a pointed homotopy colimit of a diagram whose values are all copies of $B \mathcal{F}$. It is thus natural to start looking for localizations of this universal acyclic group $\mathcal{F}$, or of $\mathcal{H}$, the "simplest" of its free summands (the definitions of these groups are given in Section 4). The main result of this paper is the following (another example of a non-perfect localization of a perfect group, based on a quotient of the same group $\mathcal{H}$, has been obtained independently by B. Badzioch and M. Feshbach in $[\mathrm{BF}]$ ).

Theorem 4.5 Let $p$ be any prime and $P$ its complementary set of primes. The Plocalization $L_{P} \mathcal{H}$ of the perfect group $\mathcal{H}$ is not a perfect group. In particular, $L_{P} \mathcal{F}$ is not perfect either.

A straightforward consequence is that the topological $P$-localization of the acyclic space $B \mathcal{F}$ is not acyclic, as its fundamental group is precisely $L_{P} \mathcal{F}$. 
Corollary 4.6 Let $p$ be any prime and $P$ its complementary set of primes. The Plocalization $L_{P} B \mathcal{H}$ of the acyclic space $B \mathcal{H}$ is not acyclic. In particular, $L_{P} B \mathcal{F}$ is not acyclic either.

This result on the first homology group contrasts with the main theorem in [CS], which deals with the first homotopy group: Any homological localization of a simply connected space is again simply connected.

The present work fits into an already large series of recent papers aimed at the study of preservation of algebraic structures by localization functors, with applications to stable and unstable homotopy theory. Let us mention the nice survey [Ca], as well as [Li], [GRS], O'S], [RST], RSV], and [CG].

Acknowledgments: We warmly thank the referee for the tremendous improvement in the organization of the material of Section 3 and 4, and in particular for his proof of Proposition 4.4. We would also like to thank Warren Dicks for pointing out Example 2.2 and Carles Casacuberta for helpful comments.

\section{Finite localizations of perfect groups}

The main result of this section could be useful to determine all finite localizations of finite simple groups, a problem which was extensively studied in [RST]. It was shown indeed in [RSV] that the localization of a finite simple group may (un)fortunately not be simple. We prove nevertheless in this section, Theorem 2.5, that all finite localizations of a non-abelian finite simple group must be perfect groups.

Let $(L, \eta)$ be any localization functor in the category of groups, where $\eta: G \rightarrow L G$ denotes the natural coaugmentation. Recall briefly that a group $G$ is called $L$-local if $\eta: G \cong L G$, and it is called $L$-acyclic if $L G=1$. Note that for every $L$-acyclic group $H$ and every $L$-local group $G$, we have $\operatorname{Hom}(H, G)=0$. We will say that a morphism

$\varphi: H \rightarrow G$ is a localization if there exists a localization functor $L$ such that $\varphi$ coincides with the coaugmentation $\eta: H \rightarrow L H$. This actually happens exactly when $\varphi$ induces a bijection $\operatorname{Hom}(H, G) \cong \operatorname{Hom}(G, G)$, see [Ca, Lemma 2.1].

Definition 2.1 A group $G$ is called almost perfect if it has no non-trivial endomorphism which factors through an abelian group, or equivalently through $G_{a b}$. 
We do not know if this property has a name in the literature, but it makes sense for any variety $\mathcal{V}$ of groups (for more about varieties, see for example [Ro, Section 1.1]). A group could be called almost $\mathcal{V}$-perfect, if it has no non-trivial endomorphism which factors through a group in $\mathcal{V}$. Of course any perfect group is almost perfect. On the other hand there are non-perfect groups which are almost perfect. Our main result Theorem 4.5 actually shows that the group $L_{P} \mathcal{H}$ is not perfect but almost perfect. Let us indicate now a more natural and elementary example of this type.

Example 2.2 In [St] Strebel considers the following central extensions of the triangle groups: $G(l, m, n)=\left\langle x, y \mid x^{l}=y^{m}=(x y)^{n}\right\rangle$. They all occur as fundamental groups of certain 3-dimensional manifolds and are finite if and only if $|l|^{-1}+|m|^{-1}+|n|^{-1}>1$. The infinite ones are Poincaré duality groups of dimension 3 (therefore torsion-free) and the abelianization can be easily computed. For example $G=G(3,8,2)$ is such a torsion-free group with $G_{a b} \cong \mathbb{Z} / 2$. It is thus almost perfect, but not perfect.

Lemma 2.3 Let $H$ be a perfect group and $H \rightarrow G$ be a localization. Then $G$ is almost perfect.

Proof. Consider an endomorphism $\psi: G \rightarrow G$ which factors through an abelian group $A$. Since $H$ is perfect, the composite $\psi \circ \eta: H \rightarrow G \rightarrow A \rightarrow G$ is trivial. On the other hand, there is a bijection of sets $\eta^{*}: \operatorname{Hom}(G, G) \cong \operatorname{Hom}(H, G)$ by the universal property of the localization. As $\eta^{*}(\psi)=0$, we infer that $\psi$ is trivial.

Theorem 2.4 Let $H$ be a perfect group and $H \rightarrow G$ be any localization with $G$ finite. Then $G$ is perfect.

Proof. Assume $G$ is not perfect. Then there exists a prime $p$ such that $\mathbb{Z} / p$ is a quotient of $G_{a b}$ because $G$ is finite. But then we would have a non-trivial endomorphism $G \rightarrow G_{a b} \rightarrow \mathbb{Z} / p \hookrightarrow G$, which contradicts the assumption that $G$ is almost perfect.

The following corollary tells us that the kind of constructions we made in the previous paper [RSV] will never yield an example of non-perfect localization of a perfect group. We have to deal with infinite groups.

Corollary 2.5 Let $H$ be a non-abelian finite simple group and $H \rightarrow G$ be any localization of finite groups. Then $G$ is perfect. 
Remark 2.6 The work of R. Göbel and S. Shelah [GS] (see also [GRS]) shows that every finite simple group admits large localizations (as large as any given cardinal). We do not know if a similar result also holds for localizations of finite perfect groups.

\section{Baumslag's class $\mathcal{P}$}

Given a set of primes $P$, a group $G$ is called $P$-local if the $p$-power map $x \rightarrow x^{p}$ is bijective for all $p \notin P$. In the notation of $[\mathrm{Ba}]$, such a group belongs to the class $D_{\omega}$. Every group $G$ admits a functorial $P$-localization $\eta_{G}: G \rightarrow L_{P} G$, i.e. $L_{P} G$ is $P$-local and for every homomorphism $\psi: G \rightarrow K$ into a $P$-local group $K$ there exists a unique homomorphism $\tilde{\psi}: L_{P} G \rightarrow K$ such that $\tilde{\psi} \circ \eta_{G}=\psi$. From now on we will invert a single prime $p$. Thus $P$ is the set of all primes different from $p$ and the localization $G \rightarrow L_{P} G$ is obtained by adding unique $p$-roots to elements without $p$-roots and identifying $p$-roots when they are not unique. In general $L_{P} G$ is a very large group, where the equation $y=x^{p}$ has a unique solution.

We shall suppose that the reader is somewhat familiar with Baumslag's work [Ba] on groups with unique roots. Another valuable reference is Ribenboim's article [Ri]. An important class of groups introduced by Baumslag in [Ba, Section 27] is the class $\mathcal{P}\left(\mathcal{P}_{\omega}\right.$ in Baumslag's notation). For a group $G$ in this class he shows that

$$
L_{P} G=\bigcup_{\alpha<\lambda} G_{\alpha}
$$

where $\lambda$ is a limit ordinal and $\left\{G_{\alpha}\right\}_{\alpha}$ is a chain of supergroups of $G$, all of them belonging to the class $\mathcal{P}$. Moreover, for every ordinal $\alpha<\lambda$, either $G_{\alpha^{+}}=G_{\alpha}$ or $G_{\alpha^{+}}$is constructed as the push-out of a diagram

$$
\mathbb{Z}[1 / p] \hookleftarrow \mathbb{Z} \stackrel{x_{\alpha}}{\longrightarrow} G_{\alpha},
$$

i.e. by adding $p$-roots to an element $x_{\alpha}$ in $G_{\alpha}$. If $\alpha$ is a limit ordinal then $G_{\alpha}=\cup_{\beta<\alpha} G_{\beta}$. This construction is explained by Baumslag in [Ba, (33.3)]. In particular groups in the class $\mathcal{P}$ are contained in their $P$-localization: $G \subseteq L_{P} G$. The importance of the class $\mathcal{P}$ comes from [Ba, Corollary 35.7], which shows that free groups belong to this class. We proceed by proving a lemma, the proof of which resembles the argument of $\mathrm{Ba}$, Theorem 33.4].

Lemma 3.1 Let $G$ be a group in $\mathcal{P}$ which is not $P$-local. Then there exists an epimorphism $L_{P} G \rightarrow \mathbb{Z}_{p^{\infty}}$. 
Proof. Since $G$ is not $P$-local there is a minimal $\alpha$ such that $G_{\alpha} \neq G_{\alpha^{+}}$. Therefore $G_{\alpha^{+}}$ is the colimit of a certain push-out diagram

$$
\mathbb{Z}[1 / p] \hookleftarrow \mathbb{Z} \stackrel{x_{\alpha}}{\longrightarrow} G_{\alpha}
$$

Construct now an epimorphism $G_{\alpha^{+}} \rightarrow \mathbb{Z}_{p^{\infty}}$ by mapping $G_{\alpha}$ trivially to $\mathbb{Z}_{p^{\infty}}$ and $\mathbb{Z}[1 / p]$ to $\mathbb{Z}_{p^{\infty}}$ by the canonical projection.

The proof goes on by a transfinite induction. Assume the epimorphism $G_{\beta} \rightarrow \mathbb{Z}_{p^{\infty}}$ has already been constructed for some ordinal $\beta>\alpha$. We need to check that it can be extended to the next step $G_{\beta^{+}}$. If $G_{\beta}=G_{\beta^{+}}$there is nothing to do, so we may assume that

$$
G_{\beta^{+}}=\operatorname{colim}\left(\mathbb{Z}[1 / p] \hookleftarrow \mathbb{Z} \rightarrow G_{\beta}\right) .
$$

A morphism out of it is determined by a pair of compatible morphisms out of $\mathbb{Z}[1 / p]$ and $G_{\beta}$. Consider the composite $\mathbb{Z} \rightarrow G_{\beta} \rightarrow \mathbb{Z}_{p^{\infty}}$. The image of $1 \in \mathbb{Z}$ generates a cyclic subgroup of order $p^{k}$ in the Prüfer group $\mathbb{Z}_{p^{\infty}}$, for some $k \geq 0$. We require now the morphism $\mathbb{Z}[1 / p] \rightarrow \mathbb{Z}_{p^{\infty}}$ to be the projection $\mathbb{Z}[1 / p] \rightarrow \mathbb{Z}[1 / p] / p^{k} \mathbb{Z} \cong \mathbb{Z}_{p^{\infty}}$. This defines the desired epimorphism $G_{\beta^{+}} \rightarrow \mathbb{Z}_{p^{\infty}}$ and the inductive process yields then an epimorphism $L_{P}(G) \rightarrow \mathbb{Z}_{p^{\infty}}$

\section{A counterexample}

Berrick and Casacuberta constructed in [BC] an acyclic two-dimensional space $B \mathcal{F}=$ $K(\mathcal{F}, 1)$ whose associated nullification functor is equivalent to Quillen's plus construction. The ideas behind its construction have been used in other contexts, for instance to show the existence of a plus-construction in the category of differential graded algebras over an operad [CRS]. The key ingredient is the universal acyclic group $\mathcal{F}$, which is a free product of an uncountable set of locally free perfect groups $\mathcal{F}_{\mathbf{n}}$ indexed by non-decreasing sequences $\mathbf{n}=\left(n_{1}, n_{2}, \cdots\right)$ of positive integers. We will make use of the group $\mathcal{H}=\mathcal{F}_{(1,1,1, \cdots)}$. The presentation of $\mathcal{H}$ is by definition

$$
\left\langle x_{1}, x_{2}, x_{3}, \ldots \mid x_{1}=\left[x_{2}, x_{3}\right], x_{2}=\left[x_{4}, x_{5}\right], x_{3}=\left[x_{6}, x_{7}\right], x_{4}=\left[x_{8}, x_{9}\right], \ldots\right\rangle
$$

so every generator is a simple commutator of two new generators. In other words, $\mathcal{H}$ is the colimit of the diagram

$$
F_{1} \stackrel{\varphi_{0}}{\longrightarrow} F_{2} \stackrel{\varphi_{1}}{\longrightarrow} F_{4} \stackrel{\varphi_{2}}{\longrightarrow} F_{8} \stackrel{\varphi_{3}}{\longrightarrow} \cdots
$$


where $F_{2^{n}}$ is a free group on generators $x_{2^{n}}, x_{2^{n}+1}, \ldots, x_{2^{n+1}-1}$ and $\varphi_{n}\left(x_{i}\right)=\left[x_{2 i}, x_{2 i+1}\right]$ for any $2^{n} \leq i<2^{n+1}$ (thus $\varphi_{n}$ is actually a free product of $2^{n}$ copies of $\varphi_{0}$ ). In general the group $\mathcal{F}_{\left(n_{1}, n_{2}, \cdots\right)}$ is defined similarly: the generators at the step $k$ are products of $n_{k}$ commutators of new generators; for more details see [BC, Example 5.3]. The rest of the section is devoted to proving that the $P$-localization of the group $\mathcal{H}$ is not perfect.

We shall need the following terminology from [MKS]. Elements of the free group $F_{n}$ on $n$ generators $x_{1}, \ldots, x_{n}$ correspond to freely reduced words $w$ in the symbols $x_{1}, \ldots, x_{n}$ : In $w$ the symbols $x_{i}^{\epsilon}$ and $x_{i}^{-\epsilon}$ never appear adjacently, where $\epsilon= \pm 1$. A word is called cyclically reduced if it is not of the form $r_{u r}^{-1}$ for some words $r$ and $u$.

Lemma 4.1 Let $F_{n}$ be the free group on $n$ generators $x_{1}, \ldots, x_{n}$ and let $w$ be a freely reduced word. If $w$ belongs to $F_{n} \backslash F_{n-1}$, then so does $w^{k}$ for any $k \neq 0$.

Proof. The proof of [MKS, Corollary 1.2.2] can be used nearly verbatim. We assume $k>0$ (the case $k<0$ will follow directly). If $w$ is cyclically reduced and contains the symbol $x_{n}$, then $w^{k}$ is freely reduced and contains thus the symbol $x_{n}$ as well for any $k \neq 0$. Moreover the freely reduced word representing $w^{k}$ starts with the same letter and ends with the same letter as $w$.

If $w$ is not cyclically reduced, it is of the form $w=r u r^{-1}$ for some cyclically reduced and non-trivial word $u$. Then $w^{k}=r u^{k} r^{-1}$, which is freely reduced since $u^{k}$ is so and starts and ends with the same symbol as $u$. Therefore $w^{k}$ contains the letter $x_{n}$ as either $u^{k}$ or $r$ contains it.

Proposition 4.2 Let $\varphi: F_{n} \rightarrow F_{2 n}$ be the group homomorphism defined by $\varphi\left(x_{i}\right)=$ $\left[x_{2 i-1}, x_{2 i}\right]$ for any $1 \leq i \leq n$. Assume $w \in F_{n}$ is a word such that $\varphi(w)=u^{k}$ for some $k \neq 0$ and some element $u \in F_{2 n}$. There exists then an element $v \in F_{n}$ such that $\varphi(v)=u$ and $v^{k}=w$. In particular, if $w$ has no p-root, then neither has $\varphi(w)$.

Proof. In [HW, Lemma 1.1], Hurley and Ward give an explicit basis for the commutator subgroup of a free group. In particular $\left[F_{2 n}, F_{2 n}\right]$ is a free group on a set of elements containing the commutators $\left[x_{2 i-1}, x_{2 i}\right]$. This shows first that $\varphi$ is a monomorphism and second that $\left[F_{2 n}, F_{2 n}\right]$ decomposes as $\varphi\left(F_{n}\right) * E$ for some free subgroup $E$ of $F_{2 n}$.

Notice next that $u$ must belong to $\left[F_{2 n}, F_{2 n}\right]$ since $u^{k}$ does so and $F_{2 n} /\left[F_{2 n}, F_{2 n}\right]=$ $\left(F_{2 n}\right)_{a b}$ is torsion free. Thus $u$ actually belongs to the image of $\varphi$ by Lemma 4.1. There exists therefore an element $v \in F_{n}$ such that $\varphi(v)=u$. As $\varphi$ is injective, $v^{k}=w$. 
Proposition 4.3 The group $\mathcal{H}$ is not P-local.

Proof. Consider the inclusion $F_{1} \hookrightarrow \mathcal{H}$. We prove that the image of $x_{1}$ in $\mathcal{H}$ has no $p$-root. It suffices to do so for the image of $x_{1}$ in $F_{2^{n}}$ for all $n \geq 1$. The proof goes by induction. Clearly $x_{1}$ has no $p$-root in $F_{1}$. The induction step is provided by Proposition 4.2

Proposition 4.4 The group $\mathcal{H}$ is in the class $\mathcal{P}$.

Proof. We want to employ [Ba, Lemma 31.1] to the expression of $\mathcal{H}$ as the colimit of

$$
F_{1} \stackrel{\varphi_{0}}{\longrightarrow} F_{2} \stackrel{\varphi_{1}}{\longrightarrow} F_{4} \stackrel{\varphi_{2}}{\longrightarrow} F_{8} \stackrel{\varphi_{3}}{\longrightarrow} \cdots
$$

By [ $\mathrm{Ba}$, Corollary 35.7] each free group $F_{2^{n}}$ is in the class $\mathcal{P}$. It suffices thus to prove that at each stage in this telescope the image $\varphi_{n}\left(C_{F_{2^{n}}}(w)\right)$ of the centralizer of any element $w \in F_{2^{n}}$ is equal to the (infinite cyclic) centralizer $C:=C_{F_{2^{n+1}}}\left(\varphi_{n}(w)\right)$ of its image.

Let $u$ be a generator of $C$. Since $\varphi_{n}(w)$ belongs to $C$ and $\varphi_{n}$ is injective, there exists some $k \neq 0$ such that $\varphi_{n}(w)=u^{k}$. Proposition 4.2 ensures now the existence of an element $v \in F_{2^{n}}$ with $\varphi_{n}(v)=u$ and $v^{k}=w$. As $\varphi_{n}$ is injective, the elements $v$ and $w$ already commute in $F_{2^{n}}$. It follows that $v \in C_{F_{2^{n}}}(w)$ and so

$$
C_{F_{2^{n+1}}}\left(\varphi_{n}(w)\right) \subset \varphi_{n}\left(C_{F_{2^{n}}}(w)\right) .
$$

The opposite inclusion is obvious, and the result follows.

Theorem 4.5 Let $p$ be any prime and $P$ its complementary set of primes. The $P$ localization $L_{P} \mathcal{H}$ of the perfect group $\mathcal{H}$ is not a perfect group. In particular, $L_{P} \mathcal{F}$ is not perfect either.

Proof. We have seen in Proposition 4.3 that the group $\mathcal{H}$ is not $P$-local, but belongs to the class $\mathcal{P}$ (Proposition 4.4). We conclude then by Lemma 3.1 that there exists an epimorphism $L_{P} \mathcal{H} \rightarrow \mathbb{Z}_{p^{\infty}}$. Thus $L_{P} \mathcal{H}$ is not a perfect group.

The group $\mathcal{F}$ contains by definition $\mathcal{H}$ as a free summand. Therefore $L_{P} \mathcal{H}$ is a retract of $L_{P} \mathcal{F}$, which cannot be perfect.

Recall that in the homotopical context $L_{P}$ denotes localization with respect to the $p$-power map $p: S^{1} \rightarrow S^{1}$. The article [CP] contains valuable information about this $P$-localization. 
Corollary 4.6 Let $p$ be any prime and $P$ its complementary set of primes. The Plocalization $L_{P} B \mathcal{H}$ of the acyclic space $B \mathcal{H}$ is not acyclic. In particular, $L_{P} B \mathcal{F}$ is not acyclic either.

Proof. By [CP, Theorem 8.7] the topological $P$-localization of a wedge of circles is given by the group theoretical one. That is, for any free group $F$, we have $L_{P} B F \simeq B\left(L_{P} F\right)$. Since $B \mathcal{H}$ is constructed as a telescope of $B F_{2^{n}}$ 's, Theorem 1.D.3 in [Dr] tells us that $L_{P} B \mathcal{H} \simeq L_{P}\left(\right.$ hocolim $\left.L_{P} B F_{2^{n}}\right)$. Therefore

$$
L_{P} B \mathcal{H} \simeq L_{P}\left(\text { hocolim } B L_{P} F_{2^{n}}\right) \simeq L_{P} B\left(\operatorname{colim} L_{P} F_{2^{n}}\right) \simeq L_{P} B\left(L_{P} \mathcal{H}\right) .
$$

The last weak equivalence is due to the fact that $L_{P} \mathcal{H}$ is isomorphic to $\operatorname{colim}_{n} L_{P} F_{2^{n}}$, this colimit being a $P$-local group (uniquely $p$-divisible) by a standard finite object argument. We conclude now that $L_{P} B \mathcal{H} \simeq B\left(L_{P} \mathcal{H}\right)$ because the latter is a $P$-local space (clearly $B G$ is $P$-local for any $P$-local group $G)$.

It could be nice to completely determine the abelianization of the $P$-localization of $\mathcal{F}$ (we just exhibited one morphism onto a Prüfer group), and also to compute explicitly the topological $P$-localization of Berrick and Casacuberta's universal space $B \mathcal{F}$.

\section{References}

[BF] Badzioch, B., Feshbach, M.: A note on localizations of perfect groups. Preprint available at http://front.math.ucdavis.edu/math.GR/0301311.

[Ba] Baumslag, G.: Some aspects of groups with unique roots. Acta Math. 104 (1960), 217-303.

[BC] Berrick, A.J., Casacuberta, C.: A universal space for plus-constructions. Topology 38 (1999), 467-477.

[Ca] Casacuberta, C.: On structures preserved by idempotent transformations of groups and homotopy types. In: Crystallographic groups and their generalizations II (Kortrijk, 1999), pp. 39-69. Contemp. Math. 262, Amer. Math. Soc., Providence 2000. 
[CG] Casacuberta, C., Gutiérrez, J.J.: Homotopical localizations of module spectra. To appear in Trans. Amer. Math. Soc..

[CP] Casacuberta, C., Peschke, G.: Localizing with respect to self-maps of the circle. Trans. Amer. Math. Soc. 339 (1993), no. 1, 117-140.

[CS] Casacuberta, C., Scherer, J.: Homological localization preserve 1-connectivity. In: Une dégustation topologique: homotopy theory in the Swiss Alps (Arolla, 1999), pp. 1-6. Contemp. Math. 265, Amer. Math. Soc., Providence 2000.

[Ch] Chachólski, W.: On the functors $C W_{A}$ and $P_{A}$. Duke Math. J. 84 (1996), no. 3, 599-631.

[CRS] Chataur, D., Rodríguez, J.L., Scherer, J.: Realizing operadic plus constructions as nullifications. To appear in K-Theory. Preprint available at http://front.math.ucdavis.edu/math.AT/0301130

[Dr] Dror Farjoun, E.: Cellular spaces, null spaces and homotopy localization. Lecture Notes in Math. 1622, Springer-Verlag, Berlin 1995.

[GRS] Göbel, R., Rodríguez, J.L., Shelah, S.: Large localizations of finite simple groups. J. Reine Angew. Math. 550 (2002), 1-24.

[GS] Göbel, R., Shelah, S.: Constructing simple groups for localizations. Comm. Algebra 30 (2002), no. 2, 809-837.

[HW] Hurley, T.C., Ward, M.A.: Bases for commutator subgroups of a free group. Proc. Roy. Irish Acad. Sect. A 96 (1996), no. 1, 43-65.

[Li] Libman, A.: Cardinality and nilpotency of localizations of groups and $G$-modules. Israel J. Math. 117 (2000), 221-237.

[MKS] Magnus, W., Karrass, A., Solitar, D.: Combinatorial group theory: Presentations of groups in terms of generators and relations. Interscience Publishers, New YorkLondon-Sydney 1966.

[O'S] O'Sullivan, N. E.: Localizations of free soluble groups, J. Group Theory 4 (2001), no. 1, 89-96. 
[Ri] Ribenboim, P.: Torsion et localisation de groupes arbitraires. In: Séminaire d'Algèbre Paul Dubreil, pp. 444-456. Lecture Notes in Math. 740, Springer-Verlag, Berlin-New York 1979.

[Ro] Robinson, D.J.S.: Finiteness conditions and generalized soluble groups. Part 1. Ergebnisse der Mathematik und ihrer Grenzgebiete 62, Springer-Verlag, New YorkBerlin 1972.

[RST] Rodríguez, J.L., Scherer, J., Thévenaz, J.: Finite simple groups and localization. Israel J. Math. 131 (2002), 185-202.

[RSV] Rodríguez, J.L., Scherer, J., Viruel, A.: Non-simple localizations of finite simple groups. Preprint available at http://front.math.ucdavis.edu/math.AT/0210405.

[St] Strebel, R.: Explicit resolutions for the binary polyhedral groups and for other central extensions of the triangle groups, Comment. Math. Helv. 58 (1983), no. 3, 433-452.

José L. Rodríguez

Área de Geometría y Topología, CITE III, Universidad de Almería, E-04120 Almería, Spain, e-mail: jlrodri@ual.es

Jérôme Scherer

Departament de Matemàtiques, Universitat Autònoma de Barcelona, E-08193 Bellaterra, Spain, e-mail: jscherer@mat.uab.es

Antonio Viruel

Departamento de Algebra, Geometría y Topología, Universidad de Málaga, Apartado de Correos 59, E-29080 Málaga, Spain, e-mail: viruel@agt.cie.uma.es 